\title{
Complexity of Work and Incident Cognitive Impairment in Puerto Rican Older Adults
}

\author{
Ross Andel, PhD ${ }^{1,2}$ Ana Luisa Dávila-Roman, PhD ${ }^{3}$ Catherine Grotz, PhD 4 \\ Brent J. Small, PhD ${ }^{1}$ Kyriakos S. Markides, PhD $^{5}$ and Michael Crowe, PhD ${ }^{6}$
}

'School of Aging Studies, University of South Florida, Tampa. ${ }^{2}$ International Clinical Research Center, St. Anne's University Hospital, Brno, Czech Republic. ${ }^{3}$ School of Public Health, University of Puerto Rico, San Juan, Puerto Rico. ${ }^{4}$ University of Liège, Psychology of Aging Unit, Liège, Belgium. ${ }^{5}$ Department of Preventive Medicine \& Community Health, University of Texas Medical Branch, Galveston. ${ }^{6}$ Department of Psychology, University of Alabama at Birmingham.

Address correspondence to: Ross Andel, PhD, School of Aging Studies, MHC 1323, University of South Florida, Tampa, FL 33620. E-mail: randel@usf.edu

Received: May 1, 2017; Editorial Decision Date: September 21, 2017

Decision Editor: Shevaun Neupert, PhD

\begin{abstract}
Objective: We investigated complexity of work in main occupation in relation to incident cognitive impairment in older Puerto Ricans.

Method: A population-based sample of 1,673 adults age 60+ for the Puerto Rican Elderly: Health Conditions (PREHCO) study was used. Cognition was measured at baseline and 4 years later using the Mini-Mental Cabán (MMC), with scoring $1.5 S D$ below the MMC score adjusted for age, education, gender, and reading ability comprising cognitive impairment. Complexity scores were derived from the 1970 U.S. Census, 1977 and 2000 Dictionary of Occupational Titles, and 2001 O*Net.

Results: Controlling for baseline age, gender, childhood economic hardship, adult money problems, depressive symptoms, and self-reported health, greater scores for most work complexity measures were associated with significantly lower risk of cognitive impairment ( $p$ s < .05), with significant odds ratios ranging between 0.74 , reflecting $26 \%$ reduction in risk for every extra standard deviation of complexity, and 0.81 . Controlling for education reduced these effects slightly but also reduced most associations to nonsignificant. The results were stronger for those with less childhood economic hardship or education $(p s<.05)$.

Discussion: Complexity of work may reduce risk of cognitive impairment in Puerto Rican older adults, especially when combined with favorable childhood economic conditions and higher educational attainment.
\end{abstract}

Keywords: Cognition, Cognitive impairment, Puerto Rico, Work complexity

Continued engagement in intellectually challenging activities has been linked to favorable cognitive outcomes in older adulthood. Work plays a major role in most people's lives. Therefore, it is no surprise that variations in exposure to work environments that reflect higher levels of intellectual challenge have been associated with better cognitive outcomes later in life observed in the United States and in Europe (Andel,
Kåreholt, Parker, Thorslund, \& Gatz, 2007; Bosma et al., 2003; Potter, Plassman, Helms, Foster, \& Edwards, 2006; Schooler, Mulatu, \& Oates, 1999). Curiously, such research is absent in Hispanic or less affluent populations. We aimed to start to fill this gap by investigating occupational complexity in main lifetime occupation in relation to cognitive outcomes among a population-based sample of older Puerto Ricans. 
We based the study on two overlapping concepts-cognitive reserve and environmental complexity. According to the concept of cognitive reserve (Costa et al., 2007; Stern, 2002), the overall use of intellectual faculties throughout life creates a stock of "cognitive capital" that delays cognitive decline with aging by improving the ability to compensate for age-related neuronal damage. Cognitive reserve is a broad concept referring to advantages with respect to brain structure and function that can promote normal cognitive function even in the presence of extensive age-related neuropathology.

The environmental complexity hypothesis (Kohn \& Schooler, 1983; Schooler, 1984) takes aim at one aspect of cognitive reserve, prolonged exposure to complex environments, as an indicator of subsequent differences in cognitive function. That is, highly complex environments that consistently offer opportunities for participating in substantively complex tasks during leisure (Schooler \& Mulatu, 2001) and at work, where they are commonly represented by complexity of work with data, people, and sometimes overall (substantive) complexity (Andel et al., 2007; Finkel, Andel, Gatz, \& Pedersen, 2009; Schooler et al., 1999) that increase intellectual flexibility and promote relatively stable cognitive functioning in older adulthood.

Although the environmental complexity hypothesis as it relates to occupation has been tested extensively, most research has been conducted in countries with relatively high socioeconomic status (SES). In addition, many of the largest epidemiologic studies of health and aging based in the United States exclude Hispanics (e.g., Howard et al., 2005), group them into one category (Bowen \& Gonzalez, 2010; Reitz, Tang, Luchsinger, \& Mayeux, 2004), or focus only on the largest subgroup, Mexican Americans (Haan et al., 2007; Markides et al., 1999; Matallana et al., 2011). Little cognitive aging research has been done in Puerto Rico, a U.S. commonwealth where the proportion of adults age $65+$ living in poverty $(39.6 \%)$ is more than triple the rate found for the rest of the United States (Aging, 2011).

The original research supporting the link between work complexity and cognition with aging was based on the measurement of complexity of work with data, people, and things, and overall (substantive) complexity either as selfreported (Schooler, 1984; Schooler et al., 1999) or occupation-based (Andel, Finkel, \& Pedersen, 2016; Andel et al., 2007; Andel, Vigen, Mack, Clark, \& Gatz, 2006; Finkel et al., 2009) constructs. More recently, additional occupation-based assessments of work complexity have been used, with similar results. For example, Smart and colleagues (Smart, Gow, \& Deary, 2014) used the 2000 Dictionary of Occupational Titles (DOT) values to measure complexity, reporting that higher complexity of work with data and people corresponded to better cognitive function at age 70, even after controlling for IQ at age 11. Even more recently, Pool and colleagues (Pool et al., 2016) used data from the 1998 Occupational Information Network (O*Net; www. onetonline.org), which is an internet-based replacement for the DOT, to measure overall work complexity. They found that this measure of work complexity, referred to as "occupational cognitive requirements score," was related to better late-life cognitive performance and slower cognitive decline. Throughout previous research into the relation between work complexity and cognitive outcomes, the idea that all measures of work complexity measure the same construct about equally has been assumed but never assessed.

Building on previous research, we posed the following research questions:

1. What is the correlation between the available measures of work complexity?

2. Is complexity of main lifetime occupation related to incident cognitive impairment among older Puerto Rican adults?

3. Do sociodemographic variables moderate any association between work complexity and cognitive impairment?

This is the first study to examine work complexity in relation to cognition in a Hispanic sample. We expected that work complexity measures would be highly correlated and that greater work complexity would be related to more favorable cognitive outcomes. Findings may vary with respect to how sociodemographic variables (gender, economic factors, and education) affect the association between work complexity and cognitive impairment. Although studies point to an association between higher work complexity and lower risk of cognitive impairment that is sustained after adjustment for childhood factors (Andel, Silverstein, \& Kåreholt, 2015; Dekhtyar et al., 2015), SES (Andel et al., 2007; Bosma et al., 2003), or education (Andel et al., 2005; Bosma et al., 2003; Kröger et al., 2008; Potter, Helms, Burke, Steffens, \& Plassman, 2007), it is less clear whether childhood conditions including education combine to magnify the association between work complexity and cognitive impairment (Dekhtyar et al., 2015) or whether work complexity may compensate for unfavorable childhood factors such as childhood socioeconomic conditions or education (Karp et al., 2009). Questions also remain regarding the potential role of gender or financial problems during adulthood in the association between work complexity and cognitive impairment.

\section{Method}

\section{Participants}

The Puerto Rican Elderly: Health Conditions (PREHCO) study is a longitudinal population-based study of older adults in Puerto Rico. The study was conducted in two waves using a probability-based sampling technique to select homes with at least one adult over 60 years of age across the entire mainland of Puerto Rico. The first wave occurred from 2002 to 2003, with the second wave occurring from 2006 to 2007. The overall response rate was 
93.9\% and there were no significant differences between those who responded and those who did not (McEnry \& Palloni, 2010). For the current study, participants were included in analyses if they reported an occupation and completed cognitive testing at baseline and at 4-year follow-up.

Overall, 4,291 participants were recruited for PREHCO; 408 were not interviewable and 3,883 (90.5\%) completed cognitive testing at baseline. Of these, 75 had incomplete data and 292 were cognitively impaired at baseline, resulting in a potential sample of 3,516 participants at baseline; 2,700 of the 3,516 had information about main lifetime occupation, of whom 2,303 reported spending at least 10 years in their main lifetime occupation, providing for a reasonable amount of exposure to the measured level of work complexity. Of these, 630 participants did not participate in the follow-up assessment, of whom 337 were dead at the time of follow-up and the remaining 293 were unreachable or unwilling to participate, leaving the analytic sample at 1,673.

\section{Measures}

\section{Cognition}

The Mini-Mental Cabán (MMC) was administered during the baseline visit and again during the follow-up visit 4 years later to assess global cognitive functioning. The MMC was designed to be more appropriate for use in Hispanic populations compared to simple Spanish translation of the Mini-Mental State Examination (MMSE) (Sánchez-Ayéndez et al., 2003). The MMC was found to have superior sensitivity and specificity compared to the MMSE for detecting clinically diagnosed dementia in a clinic-based study of older adults in Puerto Rico (SánchezAyéndez et al., 2003). Scores on the MMC can range from 0 to 20 and the MMC includes several items similar to the MMSE (orientation, immediate and delayed recall of three words, copy intersecting pentagons, and follow a threestep command), as well as items that are not part of the MMSE: visual memory (immediate recall; draw complex figure after viewing for $15 \mathrm{~s}$ ) and executive function (clock drawing, abstraction).

To determine cognitive impairment, MMC scores were regressed on years of age, female gender, years of education, and self-reported reading ability (yes/no). An expected score for each participant was calculated using the intercept and beta weights from the baseline regression model. Participants with actual MMC scores 1.5 SD or more below the predicted score were classified as having cognitive impairment.

\section{Work complexity}

Work complexity was extrapolated from self-reports of main lifetime occupation obtained during the baseline visit, and then coded based on the 2000 Standard Occupational Classification (SOC) coding scheme, which corresponds precisely to the coding used by the 2000 DOT developed by the U.S. Department of Labor. This coding is also used in an online occupational coding system, $2001 \mathrm{O}^{*}$ Net (Peterson et al., 2001), which recently replaced the paper-based DOT coding. The O*Net contains almost 300 worker characteristics created and updated through rolling administration of a questionnaire to at least 15 workers per occupation (as opposed to the DOT strategy of using observations from job analysts).

Our main measurement of work complexity uses scores from the 1970 U.S. Census, which we connected to the 2000 SOC coding scheme using code-matching tables developed by the National Crosswalk Service Center (www. xwalkcenter.org). In the 1970 U.S. Census, each occupational code was connected to the corresponding value for complexity of work with data, people, and things, as well as overall (substantive) complexity based on responses to relevant questions in large, population-based survey data (Roos \& Treiman, 1980). This measurement of work complexity has shown predictive validity using cognitive outcomes in numerous studies (Andel, 2014; Andel et al., 2005, 2006, 2015; Finkel et al., 2009; Karp et al., 2009; Kröger et al., 2008).

We also measured work complexity as follows. First, we used the 2000 DOT where digits 4-6 represent complexity of work with data (4th digit), people (5th digit), and things (6th digit), as scored by a set of qualified job analysts conducting observations of specific jobs. These scores have been used in relation to cognitive function in older adults previously (Smart et al., 2014). We also used scores for complexity of work with data and people from the 1977 DOT, which were done in the same way as for 2000 DOT. Finally, we used 10 relevant items from the O*Net, as was done previously (Pool et al., 2016), although we used the newer Version 3.1 developed in June 2001 where enough questionnaire responses were collected to make self-reports, as opposed to observations by job analysts, for the main source of information. Each complexity score was weighted by years in main lifetime occupation.

\section{Covariates}

Demographics and health were all measured at baseline during the same visit. Depressive symptoms were measured using a Spanish language version of the Geriatric Depression Scale (GDS; Yesavage et al., 1983). The commonly used 15 -item scale (GDS-15) has been shown to have acceptable reliability (Cronbach's $\alpha=.77$ ) (Friedman, Heisel, \& Delavan, 2005) and has convergent validity with other scales of depression, including the Hamilton Self-Rating Depression Scale and the Cornell Scale of Depression in Dementia (both $r s=.77$ ) (Kørner et al., 2006). The yes/no format of the GDS was designed to decrease the cognitive demand of Likert scales. The scores range from 0 to 15 , with 15 indicating the highest number of depressive symptoms.

For self-reported health, the participants were asked whether they would say that their current health was 
excellent/very good (3), good (2), average (1), or bad (0). Childhood economic hardship was assessed by summing two items about "general economic conditions in the household in which they grew up" (rated as $0=$ good, 1 = average, 2 = bad) and "economic hardship that prevented them from eating regularly, having adequate clothing or receiving medical attention" $(0=$ no, $1=$ yes $)$, with the resulting variable ranging between 0 and 3 . Money problems was measured by summing two items about having difficulties paying for goods and having difficulties paying for health care (both rated as $0=$ never, $1=$ sometimes, 2 = frequently).

\section{Analyses}

Participants were compared to dropouts using a student $t$ test for differences in means and chi-square for differences in frequencies. Descriptive statistics for the participants and correlations were used to describe the variables. Data on occupation and covariates were collected during a single baseline visit; cognitive impairment was assessed at baseline (for exclusion) and again at a follow-up visit 4 years later. In main analyses, the association between work complexity and incident cognitive impairment was assessed using binary logistic regression. This method yields odds ratios (ORs), whereby values $>1.00$ indicate increased odds of the outcome as a function of the predictor, whereas values $<1.00$ indicate reduced odds.

We considered moderation by gender, economic factors, and education for associations between work complexity and incident cognitive impairment. Results suggested that overall complexity, complexity of work with data measured with the 1970 U.S. Census and the 2000 DOT, and complexity of work with people based on the 2000 DOT provided the closest association with the odds of incident cognitive impairment based on the distance of the ORs from 1.00. Therefore, to provide a parsimonious picture of the interactions, only these complexity measures were used in the moderation analyses.

We used a bootstrapping technique developed by Hayes and colleagues to assess interaction terms (Hayes, 2012; Preacher \& Hayes, 2004). The Hayes approach implements the Johnson-Neyman technique (Johnson \& Fay, 1950), which has recently received renewed attention (Bauer \& Curran, 2005; Miller, Stromeyer, \& Schwieterman, 2013). The main advantage of this technique lies in its ability to identify the exact region in one variable where the relationship between a second variable and the outcome is statistically significant. This stands in contrast to the more widely used pick-a-point method to interpret the meaning of an interaction (e.g., selecting a point 1 SD above and below the mean).

Finally, to further examine the interrelationship between complexity and education in relation to cognitive impairment in older adulthood, we (a) used median splits to stratify the sample into those with high/low education and complexity and (b) entered the four categories (low education/low complexity [reference], high education/low complexity, low education/high complexity, and high education/high complexity) into the logistic regression models. Across the work complexity variables, around a third of the sample had the combination of low education/low complexity, another third was in the category high education/high complexity, about $20 \%$ were in the category low education/high complexity, and just over $10 \%$ were in the category high education/low complexity.

\section{Results}

There were no significant differences between the 630 who did not complete the follow-up cognitive assessment and the 1,673 who participated in any of the work complexity measures. However, those lost to dropout or death were significantly older $($ mean $=75.7$ years vs mean $=70.4$ years, $p<.001$ ), had less education (mean $=8.0$ years vs mean $=9.3$ years, $p<.001)$ and higher depressive symptomology $($ mean $=3.4$ vs mean $=3.0, p=.011)$, and were more likely to be men $(32 \%$ of men and $21 \%$ of women were lost to follow-up, $p<.001)$.

Study variables and their intercorrelations are presented in Table 1. Participants were on average about 72 years of age $(S D=8$ years), with about equal distribution of men and women, and an average of 8.6 years of education $(S D=4.8$ years $)$. About half of the sample reported unfavorable childhood economic conditions. Similarly, about half had at least some current money problems (13\% with severe money problems). Moderate to strong correlations were found between education and economic hardship variables, and between education and complexity measures, but the correlations between economic hardship variables and complexity measures were weak. Intercorrelations across complexity variables indicated especially strong correlations based on time period during which the measures were developed-the 1970s or 2000.

In the main analyses (see Table 2), we examined the association between complexity measures ( $z$-scores) and incident cognitive impairment initially controlling for demographics (age and gender), depressive symptomology, and economic factors (childhood economic hardship and money problems at baseline). One higher SD of overall work complexity was associated with about $25 \%$ lower odds of cognitive impairment, whether measured with the 1970 U.S. Census or the 2000 O*Net measure. Therefore, a person whose lifetime occupation was $1 S D$ above the mean with respect to work complexity would have around half the risk of developing cognitive impairment compared to a person with the same age, gender, economic characteristics, and depressive symptom score whose occupation was 1 SD below the mean with respect to the same measure of complexity.

With respect to work complexity with data specifically, the 1970 U.S. Census-based measure and the 2000 DOTbased measure were associated with significantly lower 


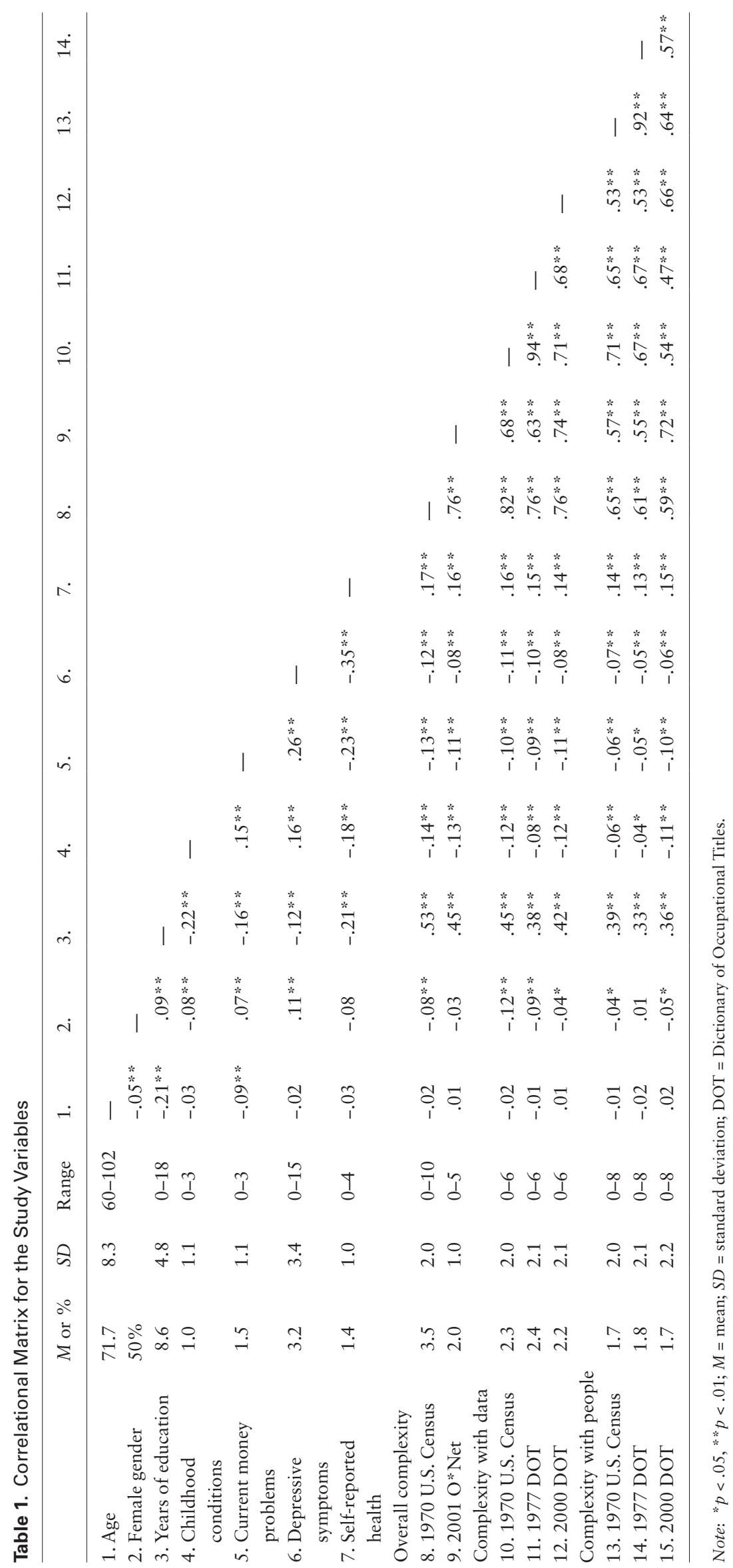


odds of cognitive impairment, by $20 \%$ and $23 \%$, respectively. Finally, work complexity with people yielded a significant association only for the 2000 DOT-based measure, whereby complexity higher by $1 S D$ was associated with odds of cognitive impairment reduced by $19 \%$. When work complexity was replaced with years of education in this adjusted model, the association between more years of education and lower odds of incident cognitive impairment was not significant $(\mathrm{OR}=0.84,95 \%$ confidence interval $[\mathrm{CI}] 0.70-1.02, p=.073)$, with the estimate suggesting that an extra standard deviation $(S D)$ of years of education would reduce the odds of cognitive impairment by $16 \%$.

When years of education was added as a covariate, only the association between the 1970 U.S. Census-based measurement of overall complexity and lower odds of cognitive impairment remained below the threshold for statistical significance, while the associations between incident cognitive impairment and the $2000 \mathrm{O} * \mathrm{Net}$ measure of overall complexity $(p=.064)$ and the 2000 DOT measure of complexity of work with data $(p=.054)$ approached significance. Of note is that including education as a covariate reduced the effects (ORs) by no more than about $20 \%$.

Subsequently, we considered moderation by gender, economic factors, and education for overall complexity, complexity of work with data based on the 1970 U.S. Census and the 2000 DOT, and complexity with people based on the 2000 DOT (the variables that yielded ORs for cognitive impairment furthest away from 1.00). In the fully adjusted models, the moderation analyses yielded no significant interactions between complexity and gender $(p s>.50)$ or money problems at baseline ( $p$ s $>.25$ ). However, the associations of overall complexity, complexity of work with data based on the 1970 U.S. Census, and complexity of work with people with incident cognitive impairment were significantly moderated by childhood economic hardship and education. Specifically, there was a significant interaction of childhood economic hardship by overall complexity measured with $2000 \mathrm{O}^{*} \mathrm{Net}(p=.036)$, complexity of work with data based on the 1970 U.S. Census $(p=.027$ ), and complexity of work with people based on the 2000 DOT $(p=.035)$ in relation to incident cognitive impairment. There were also significant interactions of education by overall complexity measured with the 1970 U.S. Census $(p=.029)$, complexity of work with data based on the 1970 U.S. Census $(p=.019)$, and complexity of work with people measured with the 1970 U.S. Census $(p=.027)$ in relation to incident cognitive impairment. See Supplementary Table 1 for all moderation results.

The statistically significant interactions are illustrated in Figures 1 and 2. Based on the Johnson-Neyman based regions of significance, participants who reported childhood economic hardship tended to not experience the same advantage in terms of reduced likelihood of cognitive impairment as a function of work complexity compared to those reporting no or only some economic hardship. Similarly, the size of association between work complexity and risk of cognitive impairment grew with more years of education.

To address the possibility that lack of variance in work complexity may have reduced the likelihood of observing significant results in participants reporting high economic hardship or low education, we generated means and SDs for each category (see Supplementary Table 2). Although mean complexity scores were lower for those reporting high economic hardship and low education, SDs indicated good variability of work complexity scores across the groups.

In sensitivity analyses where education and complexity were stratified into high/low (see Supplementary Table 3), we observed that having high education/high complexity was invariably related to significantly lower risk of incident cognitive impairment compared to having low education/ low complexity. The categories that included participants with the combination of low/high education/complexity did not differ significantly from those with low education/ low complexity.

\section{Discussion}

We set out to examine multiple measures of work complexity and relate them to incident cognitive impairment in older Puerto Ricans, offering the first look at this relationship in a Hispanic sample. Overall, we found that (a) work complexity was related to lower risk of cognitive impairment as expected, (b) most of the associations were reduced to nonsignificant when education was controlled, although the ORs for these effects changed only slightly, and (c) the association between work complexity and lower risk of cognitive impairment was mostly isolated to those who also achieved at least average education and who did not report economic hardship during childhood. Our finding that the complexity-cognitive impairment link is largely confined to those with relatively high education and at least moderately well-off financially in childhood appears to differentiate our results from those in Europe (e.g., Andel et al., 2007; Bosma et al., 2003) and the United States (Fisher et al., 2014) where the results seem generally less dependent on childhood SES or education.

The various measures of work complexity showed moderate to strong intercorrelations, with stronger correlations for measures developed during the concurrent time period. In the main analyses, after adjusting for covariates, we found all three types of complexity-overall complexity and complexity of work with data and people-to be significantly related to incident cognitive impairment. Based on these findings, it appears that the measurement of work complexity with occupation-based measures shows good stability over different measures developed in the 1970s and 2000s, possibly with the exception of DOTbased scores from 1977. Participants in this study generally entered the workforce no later than 1950s and likely exited the workforce by about 2005 (approximately when they reached age 65). 

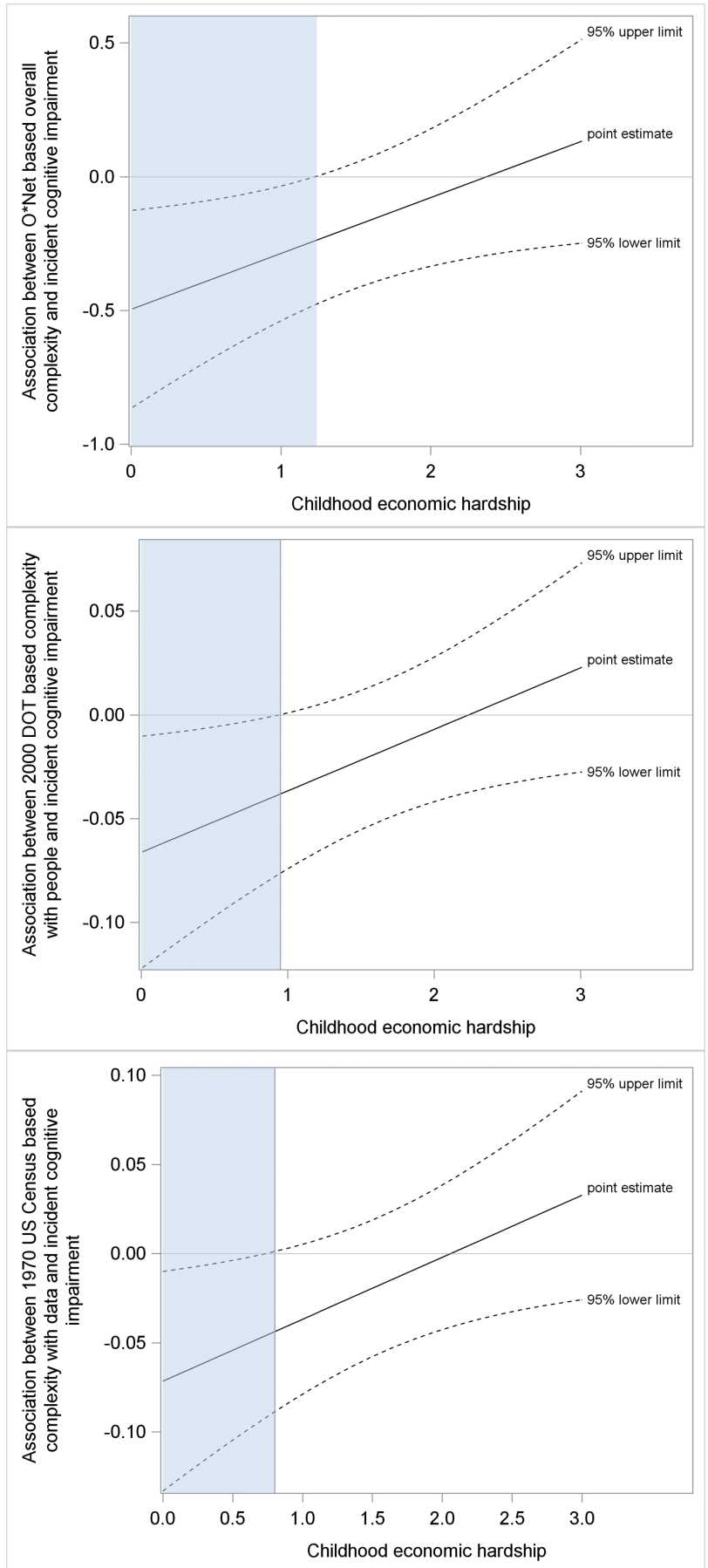

Figure 1. Association between work complexity and incident cognitive impairment with childhood socioeconomic hardship as the moderator. The region of significance is highlighted.

The finding that work complexity played a substantial protective role in late-life cognitive impairment is encouraging. It points to the possibility that engaging individuals in the workplace through assigning mentally stimulating tasks and/or providing for more freedom with respect to decision-making, both of which reflect the main characteristics of "complex" occupation (Kohn \& Schooler, 1983), may serve as an area for intervention to promote cognitive
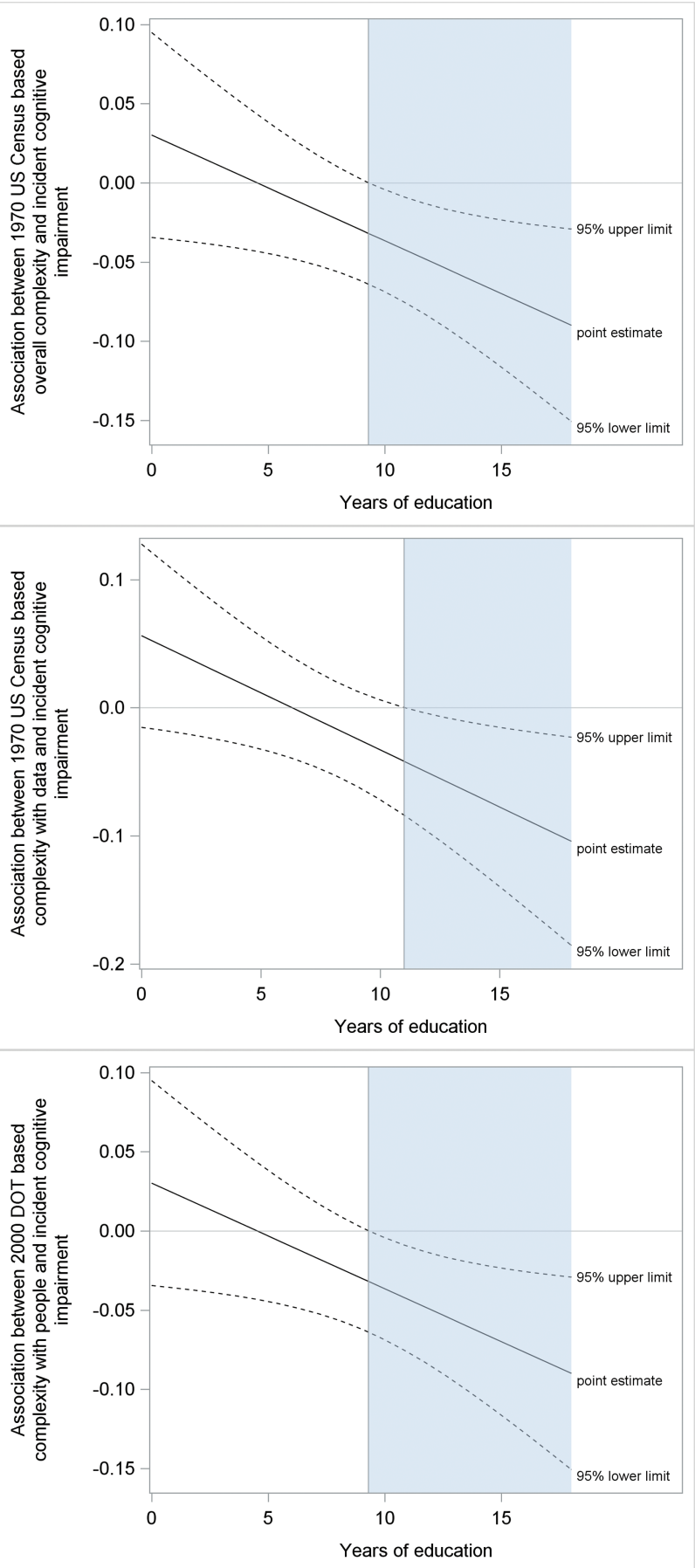

Figure 2. Association between work complexity and incident cognitive impairment with education as the moderator. The region of significance is highlighted.

health into older adulthood. Of future research interest may be both whether this effect may be generated by intervention research and, if yes, whether it may differ in a Hispanic population (e.g., be more pronounced) compared to other ethnicities.

When education was added as a covariate, only the association between overall complexity based on the 1970 U.S. Census and incident cognitive impairment still 
Table 2. Associations Between Work Complexity and Odds of Incident Cognitive Impairment

\begin{tabular}{|c|c|c|c|c|c|c|}
\hline & \multicolumn{3}{|c|}{ Model 1} & \multicolumn{3}{|c|}{ Model 2} \\
\hline & OR & $95 \% \mathrm{CI}$ & $p$ Value & OR & $95 \% \mathrm{CI}$ & $p$ Value \\
\hline \multicolumn{7}{|l|}{ Overall complexity } \\
\hline 1970 U.S. Census & 0.74 & $0.59-0.93$ & .008 & 0.76 & $0.59-0.97$ & .026 \\
\hline O*Net 2001 & 0.78 & $0.63-0.96$ & .021 & 0.80 & $0.64-1.01$ & .063 \\
\hline \multicolumn{7}{|l|}{ Complexity with data } \\
\hline 1970 U.S. Census & 0.80 & $0.65-0.99$ & .038 & 0.84 & $0.66-1.05$ & .124 \\
\hline 1977 DOT & 0.85 & $0.70-1.04$ & .114 & 0.90 & $0.72-1.11$ & .311 \\
\hline 2000 DOT & 0.78 & $0.63-0.96$ & .017 & 0.80 & $0.64-1.00$ & .053 \\
\hline \multicolumn{7}{|c|}{ Complexity with people } \\
\hline 1970 U.S. Census & 0.86 & $0.69-1.05$ & .140 & 0.90 & $0.72-1.13$ & .355 \\
\hline 1977 DOT & 0.86 & $0.70-1.06$ & .164 & 0.90 & $0.73-1.12$ & .360 \\
\hline 2000 DOT & 0.81 & $0.66-1.00$ & .054 & 0.84 & $0.68-1.06$ & .139 \\
\hline
\end{tabular}

Note $:$ OR = odds ratio; $\mathrm{CI}=$ confident interval; DOT = measurement based on the Dictionary of Occupational Titles; O*Net = measurement based on the Occupational Information Network $\left(\mathrm{O}^{*} \mathrm{Net}\right)$. Model 1 is adjusted for age, gender, childhood economic hardship, current money problems, self-rated health, and depressive symptomology; Model 2 is also adjusted for years of education.

reached the threshold for statistical significance. There are at least two plausible explanations for the effect of education on these associations. First, the association between work complexity and incident cognitive impairment may be a function of education. If true, this observation would contrast with findings from studies conducted with participants, for example, from mainland United States (Fisher et al., 2014; Potter et al., 2009), as well as from Sweden (Andel et al., 2007, 2015), the Netherlands (Bosma et al., 2003), and Germany (Then et al., 2013, 2015). In this context, education may either predetermine the range of available occupations in Puerto Rico or it may represent a stronger factor in subsequent risk of cognitive impairment than work complexity. Second, the influence of education may be more a function of a relatively weak association between work complexity and incident cognitive impairment. We observed that the point estimates approached the null result $(\mathrm{OR}=1.00)$ by only a few percentage points with the addition of education but still crossed below the threshold for statistical significance. For example, the OR for the $2001 \mathrm{O}^{*}$ Net-based measure of overall complexity was reduced from 0.77 to 0.80 -only three percentage points. Finally, it is of note that education was accounted for in the definition of cognitive impairment, which likely attenuated the effect of education in the analyses.

The moderation analyses yielded no significant interactions between complexity and gender or money problems at baseline. However, the associations of overall complexity and complexity of work with people with incident cognitive impairment were moderated by childhood economic hardship and education as illustrated in Figures 1 and 2. Specifically, the reduced incidence of cognitive impairment as a function of work complexity existed only for those who experienced no or little childhood economic hardship and those who reached at least sample average education. Therefore, the results for work complexity and incident cognitive impairment represent at least to some extent the cumulative advantage brought about by influences during childhood development. The less favorable finding was that higher work complexity was not shown to compensate for adverse childhood economic situation or low education.

The results from moderation analyses were confirmed by results from stratified analyses where education and complexity were combined to form four high/low categories. Specifically, there was a consistent relation between having the combination of high education/high complexity and a lower risk of incident cognitive impairment compared to having the combination of low education/low complexity. However, we also observed that neither high education nor high complexity was associated with reduced risk of incident cognitive impairment when combined with low scores on the other variable, suggesting that high education alone may not be protective against cognitive impairment in this sample. In fact, if any pattern of (nonsignificant) results could be discerned, it was that having low education combined high complexity and, more visibly, high education combined with low complexity conferred a somewhat elevated risk of incident cognitive impairment.

Overall, the findings that the combination of high education and high complexity provide a cumulative advantage against cognitive impairment, whereas each factor alone does not, are in contrast with results reported by Karp and colleagues with a Sweden-based sample (Karp et al., 2009), where highest levels of work complexity compensated for low education with respect to reducing the risk of dementia. However, they are in line with findings from another Sweden-based study (Dekhtyar et al., 2015) suggesting that early-life influences cannot be accounted for by complex work environment. Taken together, the results support the concept of cognitive reserve (Costa et al., 2007; Stern, 2002), whereby cognitive capital built earlier in life may provide a temporary buffer against cognitive impairment later in life. 
There are some limitations. First, our analyses included 161 cases of incident cognitive impairment, which indicates a relatively low power (the power to detect a $20 \%$ reduction in risk as statistically significant with a twotailed .05 test was $76 \%$ ). However, this may only mean that having more cases would lead to lower $p$ values. Second, not adjusting the results for multiple tests could be viewed as a limitation. However, we began the study with an evidence-based hypothesis that work complexity is associated with a reduced likelihood of cognitive impairment later in life and the multiple analyses were conducted with measures thought to reflect the same underlying construct of complexity. Third, we were unable to properly assess retirement in this sample, although the average age of the sample would indicate that most participants were either retired, very close to retiring, or worked only parttime. Finally, $630(27 \%)$ of the participants could not be assessed at follow-up, which likely affects generalizability of the results to the overall Puerto Rican population. Attrition was related to age, gender, education, and depressive symptomology.

In conclusion, we found moderate to strong correlations for various measures of work complexity, with stronger correlations for measures developed during the same time period. We also found all three types of complexity-overall complexity and complexity of work with data and people-to be related to cognitive impairment. Finally, greater work complexity significantly interacted with absence of childhood economic hardship and more education to confer greater reduction in risk of cognitive impairment, suggesting that these factors together reflect cumulative advantage that reduces the risk of cognitive impairment.

\section{Supplementary Material}

Supplementary data is available at The Journals of Gerontology, Series B: Psychological Sciences and Social Sciences online.

\section{Funding}

This work was supported in part by National Institute on Aging (NIA) Grant R21 AG045722. The content is solely the responsibility of the authors and does not necessarily represent the official views of NIA or the National Institutes of Health. C. Grotz is a Marie-Curie COFUND postdoctoral fellow at the University of Liege. Co-funded by the European Union..

\section{Conflict of Interest}

None reported.

\section{References}

Aging, A. O. (2011). A profile of older Americans: 2011. Washington, DC: Department of Health and Human Services.
Andel, R. (2014). Cognitive benefits of working. In C. Wolverson \& L. Hunt (Eds.), Work and the older person: Increasing longevity and wellbeing (pp. 89-100). Thorofare, NJ: Slack, Inc.

Andel, R., Crowe, M., Pedersen, N. L., Mortimer, J., Crimmins, E., Johansson, B., \& Gatz, M. (2005). Complexity of work and risk of Alzheimer's disease: A population-based study of Swedish twins. The Journals of Gerontology, Series B: Psychological Sciences and Social Sciences, 60, P251-P258. doi:10.1093/ geronb/60.5.P251

Andel, R., Finkel, D., \& Pedersen, N. L. (2016). Effects of preretirement work complexity and postretirement leisure activity on cognitive aging. The Journals of Gerontology, Series B: Psychological Sciences and Social Sciences, 71, 849-856. doi:10.1093/geronb/gbv026

Andel, R., Kåreholt, I., Parker, M. G., Thorslund, M., \& Gatz, M. (2007). Complexity of primary lifetime occupation and cognition in advanced old age. Journal of Aging and Health, 19, 397-415. doi:10.1177/0898264307300171

Andel, R., Silverstein, M., \& Kåreholt, I. (2015). The role of midlife occupational complexity and leisure activity in late-life cognition. The Journals of Gerontology, Series B: Psychological Sciences and Social Sciences, 70, 314-321. doi:10.1093/geronb/gbu110

Andel, R., Vigen, C., Mack, W. J., Clark, L. J., \& Gatz, M. (2006). The effect of education and occupational complexity on rate of cognitive decline in Alzheimer's patients. Journal of the International Neuropsychological Society, 12, 147-152. doi:10.1017/S1355617706060206

Bauer, D. J., \& Curran, P. J. (2005). Probing interactions in fixed and multilevel regression: Inferential and graphical techniques. Multivariate Behavioral Research, 40, 373-400. doi:10.1207/ s15327906mbr4003_5

Bosma, H., van Boxtel, M. P., Ponds, R. W., Houx, P. J., Burdorf, A., \& Jolles, J. (2003). Mental work demands protect against cognitive impairment: MAAS prospective cohort study. Experimental Aging Research, 29, 33-45. doi:10.1080/03610730303710

Bowen, M. E., \& Gonzalez, H. M. (2010). Childhood socioeconomic position and disability in later life: Results of the health and retirement study. American Journal of Public Health, 100(Suppl. 1), S197-S203. doi:10.2105/AJPH.2009.160986

Costa, D. A., Cracchiolo, J. R., Bachstetter, A. D., Hughes, T. F., Bales, K. R., Paul, S. M., ... Potter, H. (2007). Enrichment improves cognition in AD mice by amyloid-related and unrelated mechanisms. Neurobiology of Aging, 28, 831-844. doi:10.1016/j. neurobiolaging.2006.04.009

Dekhtyar, S., Wang, H. X., Scott, K., Goodman, A., Koupil, I., \& Herlitz, A. (2015). A life-course study of cognitive reserve in dementiafrom childhood to old age. The American Journal of Geriatric Psychiatry, 23, 885-896. doi:10.1016/j.jagp.2015.02.002

Finkel, D., Andel, R., Gatz, M., \& Pedersen, N. L. (2009). The role of occupational complexity in trajectories of cognitive aging before and after retirement. Psychology and Aging, 24, 563-573. doi:10.1037/a0015511

Fisher, G. G., Stachowski, A., Infurna, F. J., Faul, J. D., Grosch, J., \& Tetrick, L.E. (2014). Mental work demands, retirement, and longitudinal trajectories of cognitive functioning. Journal of Occupational Health Psychology, 19, 231-242. doi:10.1037/a0035724

Friedman, B., Heisel, M. J., \& Delavan, R. L. (2005). Psychometric properties of the 15 -item geriatric depression scale in functionally 
impaired, cognitively intact, community-dwelling elderly primary care patients. Journal of the American Geriatrics Society, 53, 1570-1576. doi:10.1111/j.1532-5415.2005.53461.x

Haan, M. N., Miller, J. W., Aiello, A. E., Whitmer, R. A., Jagust, W. J., Mungas, D. M., ... Green, R. (2007). Homocysteine, B vitamins, and the incidence of dementia and cognitive impairment: Results from the Sacramento Area Latino Study on Aging. The American Journal of Clinical Nutrition, 85, 511-517.

Hayes, A. F. (2012). PROCESS: A versatile computational tool for observed variable mediation, moderation, and conditional process modeling [White paper]. Retrieved from http://www. afhayes.com/ public/process2012.pdf.

Howard, V. J., Cushman, M., Pulley, L., Gomez, C. R., Go, R. C., Prineas, R. J., ... Howard, G. (2005). The reasons for geographic and racial differences in stroke study: Objectives and design. Neuroepidemiology, 25, 135-143. doi:10.1159/000086678

Johnson, P. O., \& Fay, L. C. (1950). The Johnson-Neyman technique, its theory and application. Psychometrika, 15, 349-367. https:// doi.org/10.1007/BF02288864

Karp, A., Andel, R., Parker, M. G., Wang, H. X., Winblad, B., \& Fratiglioni, L. (2009). Mentally stimulating activities at work during midlife and dementia risk after age 75 : Follow-up study from the Kungsholmen Project. The American Journal of Geriatric Psychiatry, 17, 227-236. doi:10.1097/ JGP.0b013e318190b691

Kohn, M. L., \& Schooler, C. (1983). Work and personality: An inquiry into the impact of social stratification. Norwood, NJ: Ablex.

Kørner, A., Lauritzen, L., Abelskov, K., Gulmann, N., Marie Brodersen, A., Wedervang-Jensen, T., \& Marie Kjeldgaard, K. (2006). The geriatric depression scale and the cornell scale for depression in dementia. A validity study. Nordic Journal of Psychiatry, 60, 360-364. doi:10.1080/08039480600937066

Kröger, E., Andel, R., Lindsay, J., Benounissa, Z., Verreault, R., \& Laurin, D. (2008). Is complexity of work associated with risk of dementia? The Canadian Study of Health And Aging. American Journal of Epidemiology, 167, 820-830. doi:10.1093/aje/kwm382

Markides, K. S., Stroup-Benham, C. A., Black, S. A., Satish, S., Perkowski, L. C., \& Oster, G. (1999). The health of Mexican American elderly: Selected findings from the Hispanic EPESE. In M. L. Wykle \& A. B. Ford (Eds.), Serving minority elders in the 21st century (pp. 72-87). New York, NY: Springer Publishing.

Matallana, D., de Santacruz, C., Cano, C., Reyes, P., SamperTernent, R., Markides, K. S., ... Reyes-Ortiz, C. A. (2011). The relationship between education level and mini-mental state examination domains among older Mexican Americans. Journal of Geriatric Psychiatry and Neurology, 24, 9-18. doi:10.1177/0891988710373597

McEnry, M., \& Palloni, A. (2010). Early life exposures and the occurrence and timing of heart disease among the older adult Puerto Rican population. Demography, 47, 23-43. https://doi. org/10.1353/dem.0.0093

Miller, J. W., Stromeyer, W. R., \& Schwieterman, M. A. (2013). Extensions of the Johnson-Neyman technique to linear models with curvilinear effects: Derivations and analytical tools. Multivariate Behavioral Research, 48, 267-300. doi:10.1080/0 0273171.2013.763567
Peterson, N. G., Mumford, M. D., Borman, W. C., Jeanneret, P. R., Fleishman, E. A., Levin, K. Y., ... Pearlman, K. (2001). Understanding work using the Occupational Information Network $(\mathrm{O} * \mathrm{NET})$ : Implications for practice and research. Personnel Psychology, 54, 451-492. doi:10.1111/j.1744-6570.2001. tb00100.x

Pool, L. R., Weuve, J., Wilson, R. S., Bültmann, U., Evans, D. A., \& Mendes de Leon, C. F. (2016). Occupational cognitive requirements and late-life cognitive aging. Neurology, 86, 1386-1392. doi:10.1212/WNL.0000000000002569

Potter, G. G., Helms, M. J., Burke, J. R., Steffens, D. C., \& Plassman, B. L. (2007). Job demands and dementia risk among male twin pairs. Alzheimer's \& Dementia, 3, 192-199. doi:10.1016/j. jalz.2007.04.377

Potter, G. G., Plassman, B. L., Burke, J. R., Kabeto, M. U., Langa, K. M., Llewellyn, D. J., ... Steffens, D. C. (2009). Cognitive performance and informant reports in the diagnosis of cognitive impairment and dementia in African Americans and whites. Alzheimer's \& Dementia, 5, 445-453. doi:10.1016/j. jalz.2009.04.1234

Potter, G. G., Plassman, B. L., Helms, M. J., Foster, S. M., \& Edwards, N. W. (2006). Occupational characteristics and cognitive performance among elderly male twins. Neurology, 67, 1377-1382. doi:10.1212/01.wnl.0000240061.51215.ed

Preacher, K. J., \& Hayes, A. F. (2004). SPSS and SAS procedures for estimating indirect effects in simple mediation models. Behavior Research Methods, Instruments, \& Computers, 36, 717-731. https://doi.org/10.3758/BF03206553

Reitz, C., Tang, M. X., Luchsinger, J., \& Mayeux, R. (2004). Relation of plasma lipids to Alzheimer disease and vascular dementia. Archives of Neurology, 61, 705-714. doi:10.1001/archneur.61.5.705

Roos, P. A., \& Treiman, D. J. (1980). DOT scales for the 1970 Census classification. In A. R. Miller, D. J. Treiman, P. S. Cain, \& P. A. Roos (Eds.), Work, jobs, and occupations: A critical review of occupational titles (pp. 336-389). Washington, DC: National Academy Press.

Sánchez-Ayéndez, M., Cabán, C. A., Fernández, L., Rosich, W., Dávila, A. L., Larriuz, M. C., ... Palloni, A. (2003). Una Escala Psicométrica Breve para Evaluar el Estado Cognitivo de Hispanoparlantes de Edad Mayor. Puerto Rico Health Sciences Journal, 22, 377-383.

Schooler, C. (1984). Psychological effects of complex environment during the life span: A review and theory. Intelligence, 8, 259-281. https://doi.org/10.1016/0160-2896(84)90011-4

Schooler, C., \& Mulatu, M. S. (2001). The reciprocal effects of leisure time activities and intellectual functioning in older people: A longitudinal analysis. Psychology and Aging, 16, 466-482. doi:10.1037/0882-7974.16.3.466

Schooler, C., Mulatu, M. S., \& Oates, G. (1999). The continuing effects of substantively complex work on the intellectual functioning of older workers. Psychology and Aging, 14, 483-506. doi:10.1037/0882-7974.14.3.483

Smart, E. L., Gow, A. J., \& Deary, I. J. (2014). Occupational complexity and lifetime cognitive abilities. Neurology, 83, 22852291. doi:10.1212/WNL.0000000000001075

Stern, Y. (2002). What is cognitive reserve? Theory and research application of the reserve concept. Journal of the International 
Neuropsychological Society, 8, 448-460. doi:10.1017. S1355617701020240

Then, F. S., Luck, T., Luppa, M., König, H. H., Angermeyer, M. C., \& Riedel-Heller, S. G. (2015). Differential effects of enriched environment at work on cognitive decline in old age. Neurology, 84, 2169-2176. doi:10.1212/WNL.0000000000001605

Then, F. S., Luppa, M., Schroeter, M. L., König, H. H., Angermeyer, M. C., \& Riedel-Heller, S. G. (2013). Enriched environment at work and the incidence of dementia: Results of the Leipzig longitudinal study of the aged (LEILA 75+). PLos One, 8, e70906. doi:10.1371/journal.pone.0070906

Yesavage, J. A., Brink, T. L., Rose, T. L., Lum, O., Huang, V., Adey, M., \& Leirer, V. O. (1983). Development and validation of a geriatric depression screening scale: A preliminary report. Journal of Psychiatric Research, 17, 37-49. doi:10.1016/0022-3956(82)90033-4 